\title{
Implementation of the Introductory Clinician Development Series: an optional boot camp for Emergency Medicine interns
}

This article was published in the following Dove Press journal:

Advances in Medical Education and Practice

25 August 2014

Number of times this article has been viewed

\author{
Alice A Min' \\ Lisa R Stoneking' \\ Kristi H Grall' \\ Karen Spear-Ellinwood ${ }^{2}$ \\ 'Department of Emergency Medicine, \\ ${ }^{2}$ Office of Medical Student Education, \\ University of Arizona, Tucson, AZ, USA
}

Correspondence:Alice A Min University of Arizona, Department of Emergency Medicine, I50I N. Campbell Avenue, Tucson, AZ 85724, USA

$\mathrm{Tel}+\mathrm{I} 520626$ I280

Email amin@aemrc.arizona.edu
Background: The transition from medical student to first-year intern can be challenging. The stress of increased responsibilities, the gap between performance expectations and varying levels of clinical skills, and the need to adapt to a new institutional space and culture can make this transition overwhelming. Orientation programs intend to help new residents prepare for their new training environment.

Objective: To ease our interns' transition, we piloted a novel clinical primer course. We believe this course will provide an introduction to basic clinical knowledge and procedures, without affecting time allotted for mandatory orientation activities, and will help the interns feel better prepared for their clinical duties.

Methods: First-year Emergency Medicine residents were invited to participate in this primer course, called the Introductory Clinician Development Series (or "intern boot camp"), providing optional lecture and procedural skills instruction prior to their participation in the mandatory orientation curriculum and assumption of clinical responsibilities. Participating residents completed postcourse surveys asking for feedback on the experience.

Results: Survey responses indicated that the intern boot camp helped first-year residents feel more prepared for their clinical shifts in the Emergency Department.

Conclusion: An optional clinical introductory series can allow for maintenance of mandatory orientation activities and clinical shifts while easing the transition from medical student to clinician.

Keywords: orientation, residency training, educational design

\section{Introduction}

The transition from medical student to first-year resident can be a time of significant stress and insecurity. Residents enter their programs with varying levels of knowledge and procedural skills.

Many residency programs provide their incoming classes with an orientation period to help prepare for their new environment and training. These orientations vary widely in educational content and duration. There is little consensus on what the ideal orientation curriculum should include. Fernandez et al described a 9-week, simulation-based, didactic lectures and skills training program for Surgery interns. ${ }^{1}$ After 4 years, this program demonstrated a statistically significant correlation between the final cognitive test upon completion of their course, and the American Board of Surgery In-Training Examination scores. Hiraoka et al implemented a 10-day orientation curriculum for first-year Obstetrics and Gynecology residents, to help them achieve baseline competence in knowledge and skills, which improved their confidence levels. ${ }^{2}$ 
There is little academic literature regarding orientation programs for residents in Emergency Medicine (EM). A survey of program directors of all EM residency programs in 1992 found that $93 \%$ of programs provided some type of orientation program. Most consisted of lectures, clinical shifts, and certification courses, such as Advanced Cardiac Life Support (ACLS). ${ }^{3}$ There was wide variation in the duration and goals of orientation from program to program. Lucas et al designed an EM orientation curriculum using the six-step Kern Model of curriculum development. ${ }^{4}$ The curriculum they developed included lectures, training on the use of evidence-based medicine, simulation and objective standardized clinical evaluations (OSCE), as well as clinical shifts in the Emergency Department (ED).

The current orientation curriculum at our residency program consists of a 4-week block made up of didactic lectures, procedural training in airway management and ultrasound, eight to ten clinical shifts in the ED, and team-building activities. Our lectures focus mainly on residency program objectives and policies, ED clinical operations, and other topics, such as professionalism and physician wellness. We have lectures on airway management and ultrasound, which are followed by time spent in the simulation lab to practice these skills with model patients. We also hold a weekend retreat for the interns, focusing on team-building activities. Prior to this initiative, we spent little time on the core content of EM. Feedback from some residents and faculty has suggested that basic EM knowledge and procedural skills should be included in the orientation. One of the main challenges was that covering additional content would require more time, detracting time from other valuable aspects of our orientation, such as ED shifts or team-building activities. Informal feedback from upper-level residents reflected that they valued the time they spent with their classmates during this block and that they learned most from seeing actual patients during ED shifts.

To preserve time spent on these aspects of orientation, we developed an optional Introductory Clinician Development series, or "intern boot camp", with a focus on core content, common presentations, and basic procedural skills. This format allowed for additional lectures and procedural time that did not detract from the clinical or team-building time during the mandatory orientation in July.

We approached the development of the intern boot camp both as an opportunity to provide support to incoming interns in their transition, and to learn from interns what their needs were and how best to meet these needs during this challenging time. Therefore, we decided that our methods needed to reflect an approach that considered stakeholders' perspectives.

"Educational design research is pragmatic because it is concerned with generating usable knowledge". ${ }^{5}$ It is a participatory design process, involving the input of stakeholders in the educational process, which has the potential to ensure the educational intervention is both responsive to learners' needs and sustainable. ${ }^{6,7}$ Taking an educationaldesign research approach to developing the intern boot camp helped to ensure the program was evidence-based as well as "flexibly adaptive to local needs", ${ }^{8}$ that is, to the anticipated needs of incoming interns, based on the self-assessed needs of the, then, current interns.

\section{Methods}

We began with a general needs assessment using SurveyMonkey ${ }^{\circledR}$ (http://www.surveymonkey.com) that was distributed to both the faculty and then current first-year residents. The survey listed several potential topics for lectures and procedure labs to include during orientation and asked participants to rate these on a scale, from "not important" to "very important". Both groups indicated that common presentations, such as chest pain and abdominal pain, sepsis, and electrocardiogram (EKG) interpretation, were "very important" to address during this series. In response to this input, we established learning goals for the boot camp series:

1. Recognize life-threatening processes in the ED

2. Learn the work-up and management of common ED presentations

3. Demonstrate basic ED procedures.

After the match results for residency programs were released, we contacted the incoming first-year residents via email to invite them to participate. Twenty-four first-year Emergency Medicine residents were eligible to participate in the boot camp. These included 15 interns in the University Campus program, six interns in the South Campus program, and three interns from the combined Emergency Medicine and Pediatrics residency program.

The boot camp was held at the end of June and was an optional series, occurring before our institution's official start date of July 1. It was scheduled for 4 hours a day, for 5 days. Each day consisted of 3 hours of lectures and 1 hour of procedure skills lab. The content and delivery of the sessions were based upon the data derived from the survey of then current interns and faculty.

We performed a postcourse survey, using SurveyMonkey, to assess whether and to what extent participants found each 
session helpful and to obtain feedback about the series as a whole. The rating scale asked participants to rate each lecture and procedure lab as "not helpful", "somewhat helpful", or "very helpful", or to indicate "not applicable" if they had not attended the session. Interns were given an opportunity to provide any comments or suggestions about how to improve the program for the coming year.

This project was reviewed and approved by the Institutional Review Board at The University of Arizona.

\section{Results}

Most of the interns participated in the series, though only six attended every session of the series. Attendance varied: 16 residents attended on day 1, 14 on day 2, 13 on day 3 , 14 on day 4, and 12 on day 5 . Reasons given from the few who did not attend any sessions were mainly scheduling conflicts with personal events.

Postcourse completion surveys were completed by 12 participants. Ten of the twelve surveys included comments. All ten remarked positively on the intern boot camp. These ten participants contributed 16 compliments to the program. They characterized it as "a great way to review important EM topics", "especially helpful", "well done", and offering "excellent and pertinent presentations". These participants also suggested a few improvements, such as adding a perspective that would compare how things are done at their hospital with practices or approaches at other sites, offering more "hands on" experiences, and providing handouts or summaries of information before or after the sessions.

Four respondents offered minor criticism of the program. For example, one respondent commented that a lecture-only format could be improved by offering hands-on experiences. Another asserted that "Five days is a lot" and suggested that boot camp be shortened to 3 days. Another said they needed more space to write comfortably when taking notes.

However, even those who added these mild criticisms recognized the program as helpful. One remarked that the work sheets provided were "super helpful", while another said that the intern boot camp "would get a million likes on FB (Facebook)". The respondent who stated, "Some lecturers were too broad and were less engaging because of less perceived applicability as well as some lecturers moving much too fast due to feeling that they had so much information to cover," also acknowledged that the "lecturers that focused on vignettes as well as the most commonly encountered items were very helpful".
This study demonstrated that conducting the intern boot camp provided much needed support for incoming residents who were recent graduates of medical school. Surveying the interns about the experience provided us with greater detail about which elements of the program were useful to orient interns to practice and which ones needed improvement. Overall, the survey enabled us to improve the program and encouraged us to continue applying an interactive design approach to this educational intervention.

Participants also were asked to rate each lecture and skills lab of the series, based on how helpful they thought each session was to their clinical preparation (Figure 1). One of the highest rated lectures was a session entitled "Introduction to the ED Patient", which discussed how to approach each patient, the basics of initial assessment and intervention, and the identification of signs of life-threatening pathology. Other sessions ranked "very helpful" by all respondents were lectures on chest pain/EKG interpretation, seizure and altered mental status, and procedural sedation. The highest rated procedure lab was the session "Central Line Insertion".

\section{Discussion}

An orientation curriculum is a common and valued aspect of residency programs. The survey by Brillman et al revealed that time during EM orientations is spent mostly in didactic lectures and certification courses. A few program administrators questioned the utility of orientation programs. There was also concern about taking time away from clinical learning and about scheduling difficulties. ${ }^{3}$ Our supplemental boot camp provided prior to the start of residency allowed for ease of scheduling shifts and the possibility of other orientation activities that our program considers valuable to the orientation curriculum.

Students have varying levels of knowledge and clinical skills when entering residency. The optional format allows first-year residents to assess their needs for learning and to participate as much, or as little, as they determine necessary.

Interestingly, our postcourse completion survey did not contain any comments expressing a desire for more time for the Introductory Clinician Development series. There was one comment suggesting we reduce the number of days from 5 to 3, while others praised the "quick" overviews and high-yield format.

There is limited statistical analysis available, given the small number of participants. Our original plan was to use the faculty clinical evaluations of first-year residents after completion of the first clinical block, comparing their 


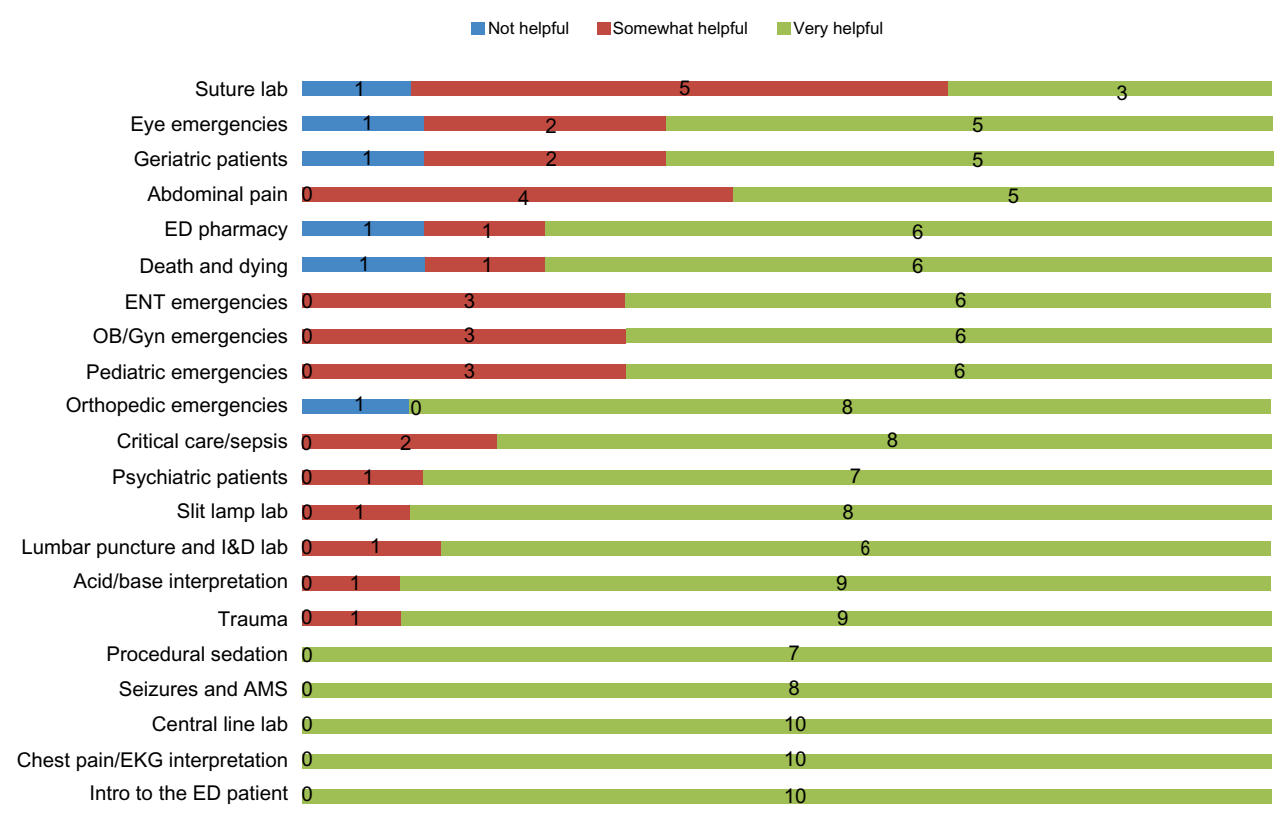

Figure I Ratings of the ICD series components, by Residents $(n=\mid 2)$.

Note: If the Resident did not attend a lecture, they marked "not applicable", which is not included in this figure.

Abbreviations: AMS, altered mental status; ED, Emergency Department; EKG, electrocardiogram; ENT, Ears, Nose, and Throat; I\&D, incision and drainage; ICD, Introductory Clinician Development; OB/Gyn, Obstetrics and Gynecology.

performance with those who did not participate. However, the majority of the incoming residents opted to participate in the series, leaving a small group for comparison. In addition, a low number of evaluations by faculty made such an analysis impractical.

However, participant feedback suggests that we should apply an educational framework known as "Before-DuringAfter". ${ }^{9}$ While this framework originated as a method to guide literacy development and comprehension, it can also be applied as a structural framework in designing educational interventions that are used to "create interactive learning environments". ${ }^{10}$

Using a Before-During-After framework will allow us to capitalize on additional opportunities for learning or reinforcing what is learned in sessions. For example, based on participant suggestions, in the next iteration we will provide overviews of material to be covered prior to attending a session. During sessions we will actively engage participants through interactive exercises, and document the questions posed. We'll also offer summaries of key points following each session, to incorporate answers to interns' questions raised during sessions, as well as include resources to facilitate further learning.

Given that only one class of interns has engaged in this experience and only one participant suggested a reduction in length of the course, at this point, we will not shorten the course. We will modify the intervention as indicated above, which incorporates suggestions repeated across comments by different participants, and determine after the next iteration of the intern boot camp whether these changes address the learning needs identified during this nascent implementation.

As this was the first year of implementation, we had a low number of participants. Thus, the generalizability of our results is limited. Future intern classes participating in this course will add important feedback to improve the series. Taking a strategic and iterative approach to designing this program has allowed us the flexibility to respond to participant suggestions and improve the program with each offering. We will also be able to analyze scores on the In-Training Examinations, to determine whether this course has an effect on these measures.

\section{Conclusion}

The format of an optional "boot camp", introduced as a supplement to orientation to present high-yield core content and basic procedural skills, provided participating firstyear residents with a well-received primer to ease their transition to clinical responsibilities. Participant interns' positive feedback has encouraged the continuation of the program. Taking an educational design research approach has helped further develop this program, which will implement participants' practical suggestions, to improve the experience. This iterative approach to designing educational interventions highlighted the importance of understanding 
how to implement educational theory in practice as well as the importance of using concrete suggestions as a driving force to further investigate educational theory, to inform future practice. The added advantage of conducting this program as an optional experience is that it allowed for a full orientation schedule without taking time from other valued activities.

\section{Acknowledgment}

The authors would like to thank the Academy of Medical Education Scholars/Office of Medical Student Education Teaching Scholars Program at the University of Arizona for their support.

\section{Disclosure}

The authors report no conflicts of interest in this work.

\section{References}

1. Fernandez GL, Page DW, Coe NP et al. Boot camp: Educational outcomes after 4 successive years of preparatory simulation-based training at onset of internship. J of Surgical Education. 2012;69(2):242-48.

2. Hiraoka M, Kamikawa G, McCartin R, Kaneshiro B. A pilot structured resident orientation curriculum improves the confidence of incoming first-year obstetrics and gynecology residents. Hawaii J Med Public Health. 2013;72(11):387-390.
3. Brillman JC, Sklar DP, Viccellio P. Characteristics of emergency medicine resident orientation programs. Acad Emerg Med. 1995;2(1): $25-31$.

4. Lucas R, Choudhri T, Roche C, Ranniger C, Greenberg L. Developing a curriculum for emergency medicine residency orientation programs. J Emerg Med. 2014;46(5):701-705.

5. McKenney S, Reeves TC. Educational design research. In: Spector JM, Merrill MD, Elen J, Bishop MJ, editors. Handbook of Research on Educational Communications and Technology. New York, NY: Springer Science and Business Media. 2014:131-140.

6. Barab SA, Luehmann AL. Building sustainable science curriculum: Acknowledging and accommodating local adaptation. Science Education. 2003;87(4):454-467.

7. Barab S, Thomas M, Dodge T, Carteaux R, Tuzun H. Making learning fun: Quest Atlantis, a game without guns. Educational Technology Research and Development. 2005;53(1):86-107.

8. Schwartz DL, Lin X, Brophy S, Bransford JD. Toward the development of flexibly adaptive instructional designs. In: Reigeluth CM, editor. Instructional-Design Theories and Models: A New Paradigm of Instructional Theory. Mahwah, NJ: Lawrence Erlbaum Associates; 1999:183-213.

9. Vacca RT, Vacca JL. Content Area Reading: Literacy and Learning Across the Curriculum. 8th ed. Boston, MA: Pearson Publishers; 2005.

10. Tuchaai T, O'Neill M, Sharplin E. Adapting to curriculum change: a theoretical framework informing the design and implementation of a curriculum intervention. English in Education. 2012;46(2):155-174.
Advances in Medical Education and Practice

\section{Publish your work in this journal}

Advances in Medical Education and Practice is an international, peerreviewed, open access journal that aims to present and publish research on Medical Education covering medical, dental, nursing and allied health care professional education. The journal covers undergraduate education, postgraduate training and continuing medical education

\section{Dovepress}

including emerging trends and innovative models linking education, research, and health care services. The manuscript management system is completely online and includes a very quick and fair peer-review system. Visit http://www.dovepress.com/testimonials.php to read real quotes from published authors. 